\title{
Challenges in Food Scientist Training in a global setting
}

\author{
ANDREAS HÖHL ${ }^{\mathrm{a}}$, GERHARD SCHLEINING ${ }^{\mathrm{a}^{*}}$, CHARLOTTE \\ HOCHGATTERER ${ }^{\mathrm{a}}$, WOLFGANG KNEIFEL ${ }^{\mathrm{a}}$, VELITCHKA GOTCHEVA ${ }^{\mathrm{b}}$, \\ ANGEL ANGELOV ${ }^{b}$, SIAN ASTLEY ${ }^{\mathrm{c}}$, AND MARIA SAARELA ${ }^{\mathrm{d}}$ \\ ${ }^{\text {a }}$ University of Natural Resources and Life Sciences Vienna, Department of Food Science and Technology, \\ Muthgasse 18, A-1190 Vienna, Austria \\ ${ }^{\mathrm{b}}$ Department of Biotechnology, University of Food Technologies, Plovdiv, Bulgaria \\ ${ }^{\mathrm{c}}$ Institute of Food Research, Norwich, UK \\ ${ }^{\mathrm{d}}$ Department of Biotechnology, VTT Technical Research Centre of Finland, Espoo, Finland \\ ${ }^{*}$ Corresponding author \\ gerhard.schleining@boku.ac.at \\ TEL: +431476546294 \\ FAX: +431476546293
}

Received: 20 January 2012; Published online: 18 October 2012

Invited paper from the $2^{\text {nd }}$ International ISEKI_Food Conference 2011

\begin{abstract}
Education and training were an integral part of the MoniQA Network of Excellence. Embedded in the "Spreading of excellence programme", Work Package 9 (Joint education programmes and training tools) was responsible for establishing a joint training programme for food safety and quality within and beyond the network. So-called 'MoniQA Food Scientist Training' (MoniQA FST) was offered to provide technical knowledge on different levels and research management skills as well. Training needs for different regions as well as for different target groups (scientists, industry personnel, authorities) had to be considered as well as developing strong collaboration links between network partners and related projects. Beside face-to-face workshops e-learning modules have been developed and web seminars were organized. In order to achieve high quality training, a quality assurance concept has been implemented. It turned out that these types of training are of high value in terms of bringing together scientists from different regions and cultures of the globe, involving highly qualified trainers as basis for a sustainable network in the future.
\end{abstract}

Keywords: training, continuous professional development, food safety 


\section{Nomenclature}

$\begin{array}{ll}\text { AOCS } & \text { American oil chemists' society } \\ \text { BOKU } & \text { University of natural resources and life sciences, Vienna } \\ \text { BRC } & \text { British retail consortium } \\ \text { BSE } & \text { Bovine spongiform encephalopathy } \\ \text { ENQ A } & \text { European network for quality assurance in higher education } \\ \text { eFST } & \text { E-learning based food scientist training } \\ \text { EU } & \text { European union } \\ \text { FP 6 } & \text { Sixth framework programme } \\ \text { FP 7 } & \text { Seventh framework programme } \\ \text { FST } & \text { Food scientist training } \\ \text { GMO } & \text { Genetically modified organism } \\ \text { HACCP } & \text { Hazard analysis and critical control points } \\ \text { IAM } & \text { Inter agency meeting } \\ \text { IFS } & \text { International food standard } \\ \text { IPR } & \text { Intellectual property rights } \\ \text { ISO } & \text { International organization for standardization } \\ \text { MoniQA } & \text { Monitoring and quality assurance } \\ \text { NoE } & \text { Network of excellence } \\ \text { QA } & \text { Quality assurance } \\ \text { PDF } & \text { Portable document format } \\ \text { WP } & \text { Work Package } \\ & \end{array}$

\section{Introduction}

The MoniQA (Monitoring and quality assurance) Network of Excellence (NoE) entitled "Towards the harmonization of analytical methods for monitoring food quality and safety in the food supply chain" was funded by the European Commission within the Sixth Framework programme Topic T5.4.5.1: Quality and safety control strategies for food (NoE). The overall aim of the project was to harmonize food quality and safety monitoring and control strategies in the food supply chain on a global scale. The international scope of MoniQA was also reflected by the core consortium which brings together 33 organizations from Europe, Africa, Middle East, Asia and New Zealand. In total, about 150 researchers from 20 countries collaborate within the MoniQA NoE. Financial support by the European commission amounts to $€ 12.3$ million. The project was carried out between February 2007 and January 2012. As a Network of Excellence, MoniQA seeks to achieve durable existence also beyond the time of EU funding in form of a non-profit association (MoniQA, 2007a).

The joint programme of activities of the MoniQA
NoE was organized in 10 work packages which were clustered into 4 major programmes (Integration, Joint Research, Spreading of excellence, Management). With respect to the large scope of the project, its international attitudes and the aim to establish a durable network, integration of and cooperation with related stakeholders played an important role. Figure 1 provides an overview on the internal project structure and stakeholder relations (MoniQA, 2007a).

The programme "Integration Activities" aimed to overcome the worldwide fragmentation in food quality and safety research. Involved work packages 1-3 sought to create expertise clusters, virtual laboratories and a durable research network by coordinated and joint use of personnel, know-how and infrastructure. MoniQA developed an internal bursary scheme and a mobility programme in order to overcome financial obstacles and legal barriers, fostering scientific exchange among different nations and also continents. The mobility and bursary programmes were accessible for scientists working for governmental and private research institutes as well as for staff employed in industry. These efforts also 


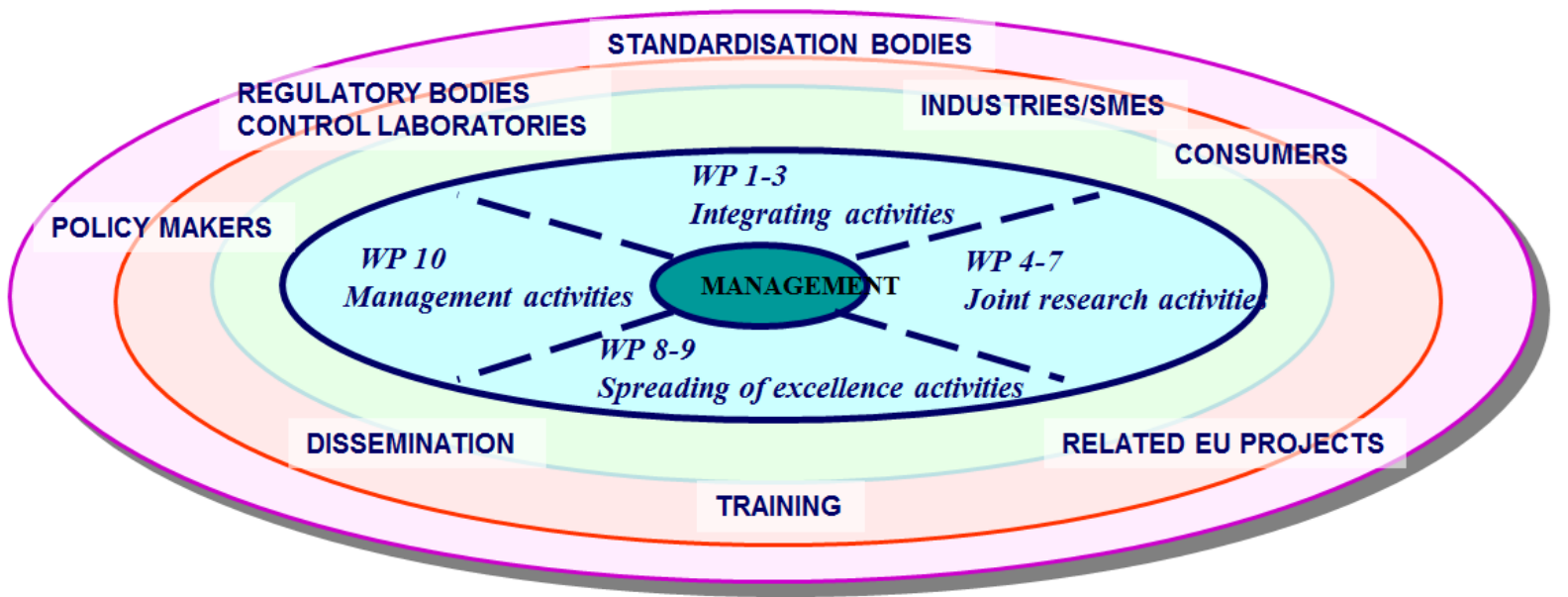

Figure 1: Structure of the MoniQA NoE (MoniQA, 2007a)

underpinned MoniQA's ambition to seek harmonized strategies also among the different groups of stakeholders (MoniQA, 2007a, 2007b).

Recent food crises like the BSE or Dioxin crisis, the GMO debate, use of new technologies (Nanotechnology) in food production and a globalized food market have upset EU citizens regarding the quality and safety of domestic and imported foods. As stated by the European commission in its White Paper on Food Safety (EC, 2000), "the European Union needs to re-establish public confidence in its food supply, its food science, its food law and its food control". Adaptations in EU law (Hygiene Package) which are mandatory to all food operators were made in order to achieve better hygiene and traceability throughout the food chain and to clearly define the responsibilities of food business operators (Hill B. 2010). In order to comply with this tightened legal framework, the demand for sophisticated detection methods and techniques is rising. Although a large variety of adequate methods and applications are available in principle, a lot of them are not accepted due to insecurities and lack of information in terms of method performance. Hence different national and international standardization organizations set up qualitative and quantitative validation protocols to prove that the method under investigation shows performance characteristics as good or better as those of the referring standard method. However, discrepancies among validation protocols lead to concerns regarding the significance of results obtained. MoniQA's Joint Research Programme pays special attention to that bottleneck by first critically analysing the current situation (needs of methods, lack of methods, high throughput methods, etc.) and subsequently by working out strategies for common harmonization, validation and implementation of new/advanced detection systems (MoniQA, 2007a, 2007c).

Training in the area of food safety, offered within and beyond the consortium, was an integral part of the network and considered as one of the key activities to facilitate knowledge sharing and learning culture within the network and to achieve harmonized levels of knowledge and skills. Embedded in the "Spreading of Excellence" programme, WP 9 - "Joint Education Programmes and Training Tools" was responsible to overcome gaps in knowledge by offering a set of closely related international training courses within and beyond the network.

\section{Materials and Methods}

In order to provide technical knowledge on different levels and research management skills for dif- 
ferent regions and for different target groups (scientists, industry personnel, authorities) methods like face-to-face workshops, e-learning and web seminars have been used. In order to achieve high quality training, a quality assurance concept has been implemented.

\subsection{Collecting information}

The work on MoniQA's training concept started with a detailed analysis of the project internal status quo which was achieved by several approaches in parallel. Most notably, a list of already existing training courses at partner organizations was established by means of an online questionnaire. Every project partner received an invitation to submit information on available courses, content, supporting teaching material, teaching language, duration, teaching methods, teaching facilities and examination mode directly online. All entered information was automatically transferred into a beforehand set up database for further processing and analysis (MoniQA, 2008a).

Besides the survey on existing courses, 2 other MoniQA internal databases have been used to set up the training concept. A database combining information of every partner organization on its applied methods, available equipment, and research facilities was used to find appropriate training sites (MoniQA, 2009a). And finally, the MoniQA expert database which summarized skills, interests and training needs of every MoniQA expert was a useful source to identify possible training topics and appropriate trainers.

MoniQA worked towards a durable international training programme. Hence, besides the internal training survey, a proper assessment on the external training situation with regard to food safety was necessary not only to avoid overlaps with already existing training, but also to identify possible collaborators who eventually could cover expertise which might not be available within the consortium. For this purpose, data was collected by means of a two-part excel based questionnaire in every country participating in the MoniQA NoE. The first part asked for any kind of organisations offering training related to food safety and quality in the surveyed country. The second part was meant to list relevant courses of all organizations defined under part one and to gather basic information on content and terms of teaching (MoniQA, 2009b).

\section{$2.2 \quad$ Face to Face Workshops}

MoniQA Food Scientist Training courses (MoniQA FST) were organized as face to face training sessions, often combining theoretical training with practical exercises. In accordance with the defined training needs and hence with the training concept, research management skills trainings were offered along with selected technical training courses. The usual duration of MoniQA FST courses was between 1 and 5 days. Participants were asked for their anonymous feedback in terms of content, quality of teaching material, trainers and overall organization of the training. A certificate was awarded to every participant at the end of the workshop.

The centrally planned annual updated training plan envisaged to offer around 10 MoniQA FSTs every year, equally distributed in Europe and Asia. Attached to selected MoniQA FSTs, at least 4 research management training courses were held every year. All workshops have been organized in a combined effort of the overall training coordinator (BOKU) and the local host. The local organizer was responsible to deliver a draft budget, an agenda and had to organize on site accommodation and conference and laboratory facilities needed during the workshop. Once the budget and the agenda met the requirements of MoniQA, the training administrator gave permission to the local organizer to implement the workshop and dissemination was started on the national and international level. Registration and recovery of workshop fees was administered centrally by the training administrator. Only in exceptional cases, cash payment on site was possible. At some occasions, MoniQA FSTs have been organized in cooperation with related EU projects like HealthGrain (http://www.healthgrain.org/) or MycoRed (http://www.mycored.eu/) or with organizations like AOCS/IAM (http://www.aocs.org/Methods/content.cfm 
Food Scientist Training |117

?ItemNumber=2050). In such cases, the financial load was shared between the two organizers and dissemination was done using the distribution channels of both networks. In return, experts from both organizations benefited from free registration.

\section{$2.3 \quad$ E-learning}

One benefit of the MoniQA NoE was the global pool of experts, enabling the consortium to deliver training in a great variety of topics all around the world. However, such an internationalized training approach required a great deal in time and money for trainers and trainees. In order to address these difficulties, MoniQA started to implement e-Learning. The open source teaching platform "Moodle" was selected due to its relative high use in academic institutions. MoniQA's adapted e-Learning platform featured password protected access and was split into an introductory section and an actual training section.

The introductory section was meant to assist inexperienced trainers and trainees by a set of guidelines, course templates and forums. As a supportive measurement face to face workshops on the proper use of "Moodle" were offered one time in Europe (Vienna, Austria) and two times in Asia (Hanoi, Vietnam and Chengdu, China). The ulterior motive of these training courses was to overcome knowledge wise barriers and to kick start the e-Learning programme by explaining the principles of e-Learning and by highlighting and explaining possibilities and functional principles of the Moodle system in form of case studies (MoniQA, 2010a).

The second part of the platform, the training section, contains all MoniQA e-Learning courses. MoniQA e-Learning courses are open to everyone, although MoniQA associated partners and external organizations have to pay a small fee. Each course featured self study material, self tests and forums. The overall maintenance of the platform, technical support and registrations are handled centrally by the e-learning coordinator (BOKU). Content, tasks and communication with students during the course was within the responsibility of the MoniQA partner organiza- tion that created the course. IPR on the training content and materials stayed with the course creator (MoniQA, 2010a).

Trainees participating in any of these eFSTs have 2 months to complete the course, starting from the day of enrolment. During the course, students were able to access the training material anytime and anywhere via web browsers. Every course has 1 person for content-related problems and one person for technical problems who could be contacted by the participants via forums or email. Once the trainees accomplished all tasks set by the trainer, they were awarded a certificate.

\subsection{Web Seminars}

In order to run web seminars, several systems have been tested. The selected web seminar licence (CITRIX, UK, www.gotomeeting.co.uk) enabled the consortium to offer web seminars to 100 participants at a time. Technically, a MoniQA web seminar was conducted by audio streaming, video streaming and desktop sharing. Questions to the speaker were addressed via audio connection (the use of a headset was recommended) or written in a chat box. At the end of a web seminar online feedback from participants was collected anonymous by an online evaluation. The presenter of a web seminar only had to prepare a power point presentation and to perform a test run with the system a few days before the web seminar. Registration, dissemination and technical support was within the responsibility of the training administrator. Potential presenters were asked to submit an application form with information about the topic, the presenter, date and time, etc. in order to schedule and advertise the web seminar mainly by email and newsletters.

A workflow was elaborated as an agenda for carrying out the web seminar. An administrator introduced the agenda and explained how participants can ask questions. After introducing the topic and the presenter, the control was given to the presenter. The presenter opened his presentation on her/his computer and shared the screen with the participants. Further discussions were enabled by raising a virtual hand, asking for the 
microphone, which could be switched on and off for each attendee.

\subsection{Bursary Scheme}

The MoniQA Exchange and Mobility Programme was developed in order to overcome one of the major drawbacks in international training - money. It was expected that exchange of staff within the consortium and attendance of MoniQA partners in MoniQA FSTs will substantially influence the extent of knowledge exchange within the consortium. Funding personnel exchange and financial support for attending conferences are benefits often available for senior staff only. MoniQA on the opposite provided financial support also to junior staff and $\mathrm{PhD}$ students.

Funding was eligible for attending MoniQA conferences, MoniQA training, personnel exchange among partner organizations and in certain cases, attendance at external conferences has been supported. Applications for bursaries, including a short statement why the applicant personally but also the involved organizations will benefit, had to be submitted to the Mobility and Exchange coordinator who awarded the bursaries on a competitive basis. Two types of bursaries were available. The full bursary covered all costs, whereas travel bursaries only covered travel expenses and course fees. In terms of full bursaries, the whole amount was only paid off if the beneficiary submitted a training report (MoniQA, 2009c).

\subsection{Quality Assurance for training}

The developed Quality Assurance (QA) concept has principally taken into consideration the European context and demands, and is based on the internationally developing processes and experience in this area. Therefore, the development of the QA concept is based mainly on the European practice, which has taken into account the key international experiences and initiatives, and more specifically, the standards and guidelines developed by ENQA, which are also in line with the principles of quality management of ISO 9001 (2008). The main basic principles of this concept are customer focus, leadership, involvement of people, process approach and continual improvement. The ENQA standards and the criteria for quality assessment of MoniQA training are listed in Table 1 (ISO 9001, 2008; ENQA, 2009; MoniQA, 2012a).

\section{Results and Discussion}

Training needs for different regions as well as for different target groups (scientists, industry personnel, authorities) had to be considered as well as developing strong collaboration links between network partners and related projects. So-called 'MoniQA Food Scientist Training' (MoniQA FST) was offered, which provides technical knowledge on different levels and research management skills as well. Beside faceto-face workshops e-learning modules have been developed and web seminars were organized. In order to achieve high quality training, a quality assurance concept has been implemented.

\subsection{Training needs}

In accordance with the overall aims of MoniQA, the training concept was expected to overcome legal, time-wise, linguistic and financial barriers and to consider training needs and gaps of different regions and different target groups. MoniQA aimed at delivering tailor made training to several different target groups (industry, research), at different levels (senior staff, junior staff) which reflects local needs as well as personnel needs and which finally is easily accessible. An overview on identified training needs is given in Table 2 .

Hence, the selected approach was a module based training concept, made up of several short term courses (1-5 days), E-learning and web seminars, which was flexible enough to consider above mentioned aspects and also enabled the consortium to offer training related to recent events (food crises, new legislation, etc.) within a relative short time. Knowledge gaps within the network were addressed by first defining existing knowledge and training needs at each partner institution and then selecting topics, trainers and destinations for training courses. Supported by the mobility and bursary schemes and 
Food Scientist Training |119

Table 1: ENQA standards and the criteria for Quality Assurance of MoniQA training (ENQA, 2009; MoniQA, 2012a)

\begin{tabular}{|c|c|c|}
\hline $\begin{array}{l}\text { ENQA Standard } \\
\text { MoniQA principle }\end{array}$ & Contents & MoniQA criteria \\
\hline \multirow[t]{5}{*}{$\begin{array}{l}\text { 1.Policy and proce- } \\
\text { dures for quality as- } \\
\text { surance }\end{array}$} & $\begin{array}{l}\text { Policy and associated procedures for } \\
\text { the assurance of the quality of MoniQA } \\
\text { FST (workshops, courses) }\end{array}$ & $\begin{array}{l}\text { The consortium organises and carries out } \\
\text { training in accordance to its goals and ob- } \\
\text { jectives. }\end{array}$ \\
\hline & \multirow[t]{3}{*}{ Interactions consortium members. } & $\begin{array}{l}\text { The consortium supports training activi- } \\
\text { ties of its member organisations. }\end{array}$ \\
\hline & & $\begin{array}{l}\text { MoniQA disseminates training opportuni- } \\
\text { ties and implements novelty research re- } \\
\text { sults in its courses. }\end{array}$ \\
\hline & & $\begin{array}{l}\text { MoniQA QA group develops an internal } \\
\text { system for evaluation, maintenance and } \\
\text { improvement of FST quality. }\end{array}$ \\
\hline & $\begin{array}{l}\text { Strategy and policy for the continu- } \\
\text { ous enhancement of quality. The strat- } \\
\text { egy, policy and procedures should have } \\
\text { a formal status and be publicly avail- } \\
\text { able. They should also include a role } \\
\text { for trainees and other stakeholders. }\end{array}$ & $\begin{array}{l}\text { The consortium publicly presents its mis- } \\
\text { sion, policy and strategy. }\end{array}$ \\
\hline $\begin{array}{l}\text { 2.Approval, moni- } \\
\text { toring and periodic } \\
\text { review of training } \\
\text { courses }\end{array}$ & $\begin{array}{l}\text { MoniQA should have formal mecha- } \\
\text { nisms for the approval, periodic review } \\
\text { and monitoring of workshops and train- } \\
\text { ing courses offered. }\end{array}$ & $\begin{array}{l}\text { MoniQA QA group elaborates and ob- } \\
\text { serves procedures for development, ap- } \\
\text { proval, monitoring and improving of FST. }\end{array}$ \\
\hline $\begin{array}{l}\text { 3.Assessment of } \\
\text { trainees }\end{array}$ & $\begin{array}{l}\text { Trainees should be assessed using pub- } \\
\text { lished procedures which are applied } \\
\text { consistently. }\end{array}$ & $\begin{array}{l}\text { The QA group develops and publishes } \\
\text { procedures for the assessment of trainee's } \\
\text { knowledge and skills acquired from } \\
\text { MoniQA training. }\end{array}$ \\
\hline \multirow[t]{2}{*}{$\begin{array}{l}\text { 4. Quality assurance } \\
\text { of teaching staff }\end{array}$} & \multirow[t]{2}{*}{$\begin{array}{l}\text { MoniQA should have ways of ensuring } \\
\text { that staff involved in the FST is quali- } \\
\text { fied and competent to do so. }\end{array}$} & $\begin{array}{l}\text { The consortium ensures the professional } \\
\text { level, qualification and development of its } \\
\text { trainers. }\end{array}$ \\
\hline & & $\begin{array}{l}\text { The consortium collaborates with non- } \\
\text { MoniQA organizations when necessary. }\end{array}$ \\
\hline \multirow[t]{3}{*}{$\begin{array}{l}\text { 5.Learning re- } \\
\text { sources and trainee } \\
\text { support }\end{array}$} & \multirow[t]{3}{*}{$\begin{array}{l}\text { MoniQA should ensure that the re- } \\
\text { sources available for the training activ- } \\
\text { ities are adequate and appropriate for } \\
\text { each course offered. }\end{array}$} & $\begin{array}{l}\text { Each training organisation manages and } \\
\text { develops the infrastructure, technical and } \\
\text { information resources necessary for carry- } \\
\text { ing out the suggested course }(\mathrm{s}) \text {. }\end{array}$ \\
\hline & & $\begin{array}{l}\text { The training organisations may collaborate } \\
\text { with other universities and organisations. } \\
\text { MoniQA attracts trainees and aids their } \\
\text { professional realization }\end{array}$ \\
\hline & & $\begin{array}{l}\text { The consortium disseminates training op- } \\
\text { portunities. }\end{array}$ \\
\hline $\begin{array}{l}\text { 6.Information sys- } \\
\text { tems }\end{array}$ & $\begin{array}{l}\text { MoniQA should collect, analyse and } \\
\text { use relevant information for the effec- } \\
\text { tive management of its FST. }\end{array}$ & $\begin{array}{l}\text { The QA group has elaborated adequate } \\
\text { organization for management of MoniQA } \\
\text { FST. }\end{array}$ \\
\hline $\begin{array}{l}\text { 7.Public } \\
\text { tion }\end{array}$ & $\begin{array}{l}\text { MoniQA should regularly publish up } \\
\text { to date information, both quantita- } \\
\text { tive and qualitative, about the training } \\
\text { courses offered. }\end{array}$ & $\begin{array}{l}\text { The consortium actively promotes training } \\
\text { opportunities offered and disseminates rel- } \\
\text { evant information. }\end{array}$ \\
\hline
\end{tabular}

\begin{tabular}{l|l|l|l} 
IJFS & October 2012 |Volume 1 pages 114-125
\end{tabular} 
Table 2: Training needs in three MoniQA relevant regions. + ..No/minor needs, ++ ..needs, +++ ..major needs.

\begin{tabular}{llll}
\hline \multicolumn{1}{l}{ Region: } & $\begin{array}{l}\text { China, Vietnam, } \\
\text { Indonesia }\end{array}$ & EU members & Egypt, Turkey \\
\hline Type of training & & & \\
Face to face & + & + & + \\
e-learning & +++ & ++ & +++ \\
Web seminars & +++ & ++ & ++ \\
Short term courses & +++ & ++ & ++ \\
International courses in English & +++ & & +++ \\
Target Groups & & ++ & \\
Courses for industry & & ++ & ++ \\
Courses for academia & +++ & ++ & ++ \\
Content & ++ & & \\
Research management skills & & +++ & +++ \\
Food quality / Food safety & +++ & + & ++ \\
Food chemistry & +++ & ++ & ++ \\
Food microbiology/molecular biology & ++ & ++ & ++ \\
Food technology & + & + & + \\
\hline
\end{tabular}

in combination with E-learning and web seminars, the MoniQA training programme successfully overcame training barriers and facilitated knowledge transfer among the continents and different stakeholders.

It is generally accepted that many European researchers are still lagging behind in soft skills, which are generally under-emphasized in most of the curricula. Research Management includes scientific communication skills such as scientific writing, designing and presenting posters and flyers, presentation skills, proposal preparation, management of research projects, research budget planning and administration. Therefore it was decided to offer research management training courses within the network to improve the general skills set of the researchers. It is also planned to extend the portfolio of research management skills and to consider IPR, EU policy making and socio-economic aspects.

\subsection{What is available within the NoE}

All courses were first grouped according to their subject and then those not really related to the scope of MoniQA (Food Safety, Food Quality) have been ruled out. Content-wise, most aspects of food safety and some quality aspects were covered. The majority of courses were focused on food technology and food chemistry, but courses in hygiene, microbiology, economy, legislation, molecular biology and nutrition were also carried out (Figure 2).

In depth analysis of the remaining courses made it evident that only a low number of the courses would comply with the needs of the envisaged training concept. First of all, most of the courses were University courses and ran as long term courses, lasting several weeks to months without any support of E-learning or digital teaching materials. On the other hand, only $13 \%$ of the courses were offered completely in English language, 28\% as a combination of English and national language and the majority of courses $(46 \%)$ were available in national language only. 
The most common teaching method is face to face, followed by practical trainings, seminars and group works (MoniQA, 2008b). E-Learning plays just a minor role so far, but would be of great interest for offering education on an international level in the future. Figure 3 depicts the different teaching methods and to what extent they are used by consortium partner.

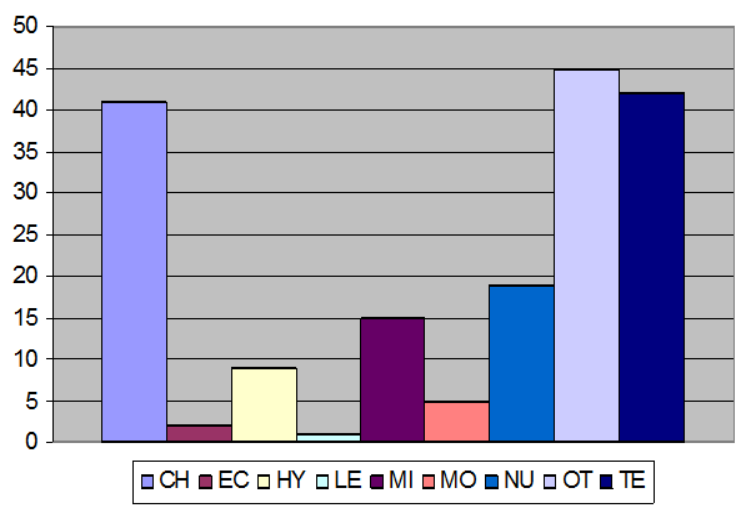

Figure 2: Areas and numbers of training courses available within the MoniQA NoE (MoniQA, 2008b). CH..Chemistry, EC..Economy, HY..Hygiene, LE..Legislation, MI..Microbiology, MO...Molecular biology, NU..Nutrition, TE..Technology, OT..Other.

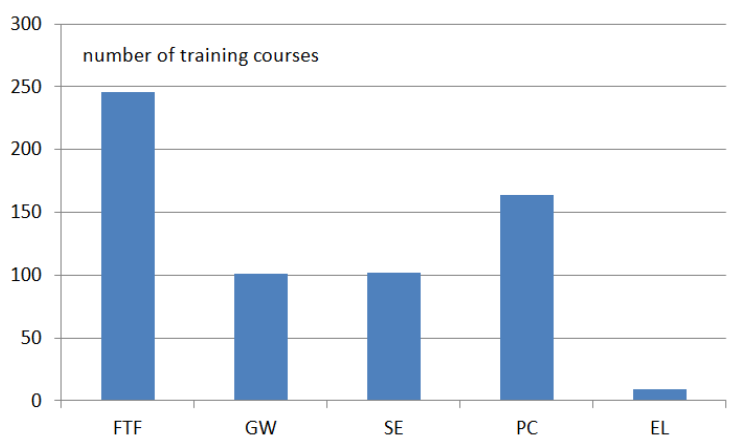

Figure 3: Extent of teaching methods used by the partners of the MoniQA NoE (MoniQA, 2008b) FTF..Face to face, GW..Group work, SE..Seminars, PC..Practical class, EL..e-learning.

\subsection{What training is offered beyond the NoE}

Collected information was analyzed and fed into the database containing already information on internal training. Valid data was available from Austria, Belgium, Bulgaria, China, Egypt, Germany, Greece, Hungary, Italy, Spain, Turkey and Vietnam. With the exception of Vietnam, in all countries most of the external training was about food safety in the broadest sense. Especially principles and implementation of HACCP and quality standards such as ISO, IFS and BRC were among the most common topics. A considerable number of courses were also offered in the areas of food chemistry, food microbiology and food technology. In line with findings drawn from the survey on internal courses, the number of research management skill courses was again very low - only $2 \%$ of all courses. Relative and absolute comparison of the teaching languages was not appropriate, as the number of submitted courses varied among the different countries. But it was evident that most of the courses were offered in the national language. The vast majority of the evaluated courses were offered as short term courses, lasting between 1 and 5 days.

Upon completion, the combined database on internal and external training offered information on a little more than 300 internal and $800 \mathrm{ex}$ ternal courses plus the providing organizations. Figure 4 shows the used training resources within and beyond MoniQA.

\subsection{Development of a training concept}

After analyzing the training needs (3.1) and what is available within (3.2) and beyond the network (3.3), the following topics have been selected for face to face workshops and e-learning courses: food microbiology, food chemistry, food authenticity, molecular biology, food safety, HACCP, hygienic design, cleaning and cleaning validation, quality assurance, project management and scientific communication.

For course organizers and content providers standard operation procedures (SOPs), application forms, templates to calculate the budget and for 


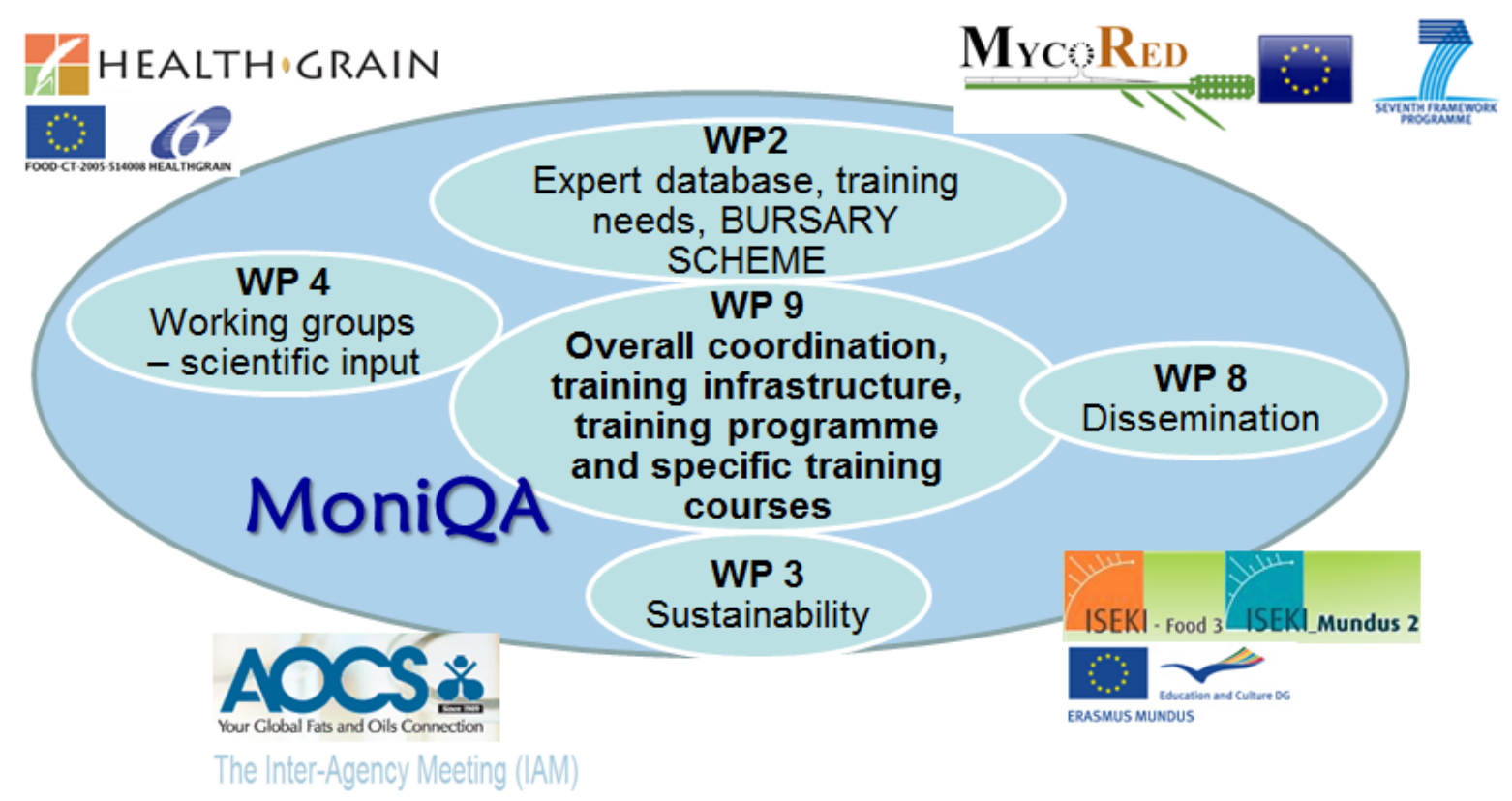

Figure 4: Used training resources within and beyond MoniQA.

power point presentations as well as for an elearning course have been developed and made available for download. In addition, guidelines on how to use the e-learning platform have been made available.

\subsection{Face to Face Workshops}

The first MoniQA FST was offered in November 2007. Till July 2011, 39 MoniQA FSTs have been offered in 12 countries (Austria, Bulgaria, China, Czech Republic, Hungary, Indonesia, Italy, Malaysia, New Zealand, Turkey, United Kingdom and Vietnam) and delivered knowledge to an audience composed of 26 different nationalities in total. At 12 workshop occasions research management training was attached. Content-wise, priority was given to chemical contaminants (heavy metals, polychlorinated biphenyls, pesticides), microbiological methods (rapid alternative methods, PCR), allergens (Analysis, risk assessment) and Mycotoxins (safe food trade, Fusarium toxins in cereal, Mycotoxins in fruits), food authenticity, reference materials and method validation. Further topics covered within the training programme were dealing with socio economic impacts of food regulations, hygienic design, cleaning, cleaning validation, shelf life determination, food indexing, HACCP and risk management. In terms of research management training, the focus was on communication, project development, project management and e-Learning.

\subsection{E-learning}

At the end of the project, the MoniQA eLearning programme comprised five fully functional e-Learning courses developed by Austrian, Italian and Indonesian organizations. Language barriers have been addressed by multilingual approaches. For example, one course is available in seven languages (English, Italian, Bulgarian, Hungarian, Turkish, Arabic, and Vietnamese). For the design of a course and also to reach potential users, it turned out that the proper formulation of learning outcomes - what the participants will know and are able to do after completion of a course - was extremely important and helpful.

During the development of a course the availability of many experts from the network for eval- 
Food Scientist Training |123

uation and for contributions could assure highquality content. E-content was mainly provided as pdf files, generated from powerpoints, in order to avoid incompatibilities. With pdf as format the participants do not need to have a licence for the different programmes with which the material was created. As E-tivities, discussion forums were mainly used to assign tasks and collect reports. For self assessment and the final exam quizzes were used (Salmon, G. 2002).

During an internal evaluation phase, technical problems occurred due to limited bandwidths in Asian countries and due to different browser settings. Several browsers have been checked as to what extent they cause troubles with different Moodle features.

E-learning could also be used to support Face to Face Workshops by providing study material, to prepare the workshop and to support further discussions and activities of the participants and to involve people from other countries. The courses can also be used for blended learning, which means that interactive parts are moderated by trainers within predefined timeslots and combined with face-to-face workshops.

\subsection{Web Seminars}

Web seminars were the most flexible tool within the training concept. They can be prepared in a relative short time and at low cost, still facilitating live communication between presenter and attendees. Thus, web seminars have been used whenever training had to be developed within a short time - for instance in the aftermath of a sudden food crisis like the Austrian Listeria case in 2009/2010 (MoniQA, 2012b).

\subsection{Quality Assurance}

Standard operating procedures (SOP's) and forms have been prepared to carry out and document the quality assurance of training. The management structure and responsibilities as well as the pricing policy have to be specified. It was written down how to make an annual plan, how to design and develop a course, how to advertise, how to carry out a course, how to assess the participants and how to improve the quality as- surance itself.

In a first step to implement above listed procedures, the responsibilities within the task force have been identified. The training coordinator was in charge of the overall coordination of activities and had to prepare the annual training plan, considering training needs and make sure that the finances comply with the budget allocated by the financial manager. According to the SOPs', the training coordinator sent the annual draft training plan to all task force members and subsequently reviewed and incorporated partner views. The adapted annual plan was then discussed with the financial management and the total annual budget reserved for training was allocated. In terms of new training courses, a planning team supervised the course development. Dissemination was performed under the aegis of the training coordinator and the training dissemination manager with support from the organizers on site. A common pricing policy was defined by the training coordinator in cooperation with financial manager and all task force members (MoniQA, 2012a). After the training coordinator gave the final permission, the realization team, consisting of local organizer, coordinator and dissemination manager, started with the implementation of the training.

At the end of every MoniQA branded training course, the realization team had to perform an evaluation of the training based on participant feedback. A summarized evaluation of all training courses offered between February 2011 and January 2012 is depicted in Table 3.

\subsection{Problems encountered}

The development of MoniQA training relied to a large extent on the information gathered by the consortium. Huge efforts have been made in the first round to set up the database on internal and external training and to establish rules dedicated to maintain and update the data. However, these rules proved to be insufficient and impracticable for the simple reason that man months were reallocated to other tasks and hence partners had only limited resources left to maintain the data. Especially important for selecting meaningful training topics was the expert database which 
124 | Höhl et.al.

Table 3: Summarized feedback obtained by MoniQA training participants between February 2011 and January 2012.

\begin{tabular}{|c|c|c|c|c|c|c|}
\hline & $\begin{array}{c}\text { Very } \\
\text { satisfied }\end{array}$ & Satisfied & Neutral & Dissatisfied & $\begin{array}{c}\text { Very } \\
\text { dissatisfied }\end{array}$ & Total \\
\hline Overall quality of the event & 150 & 61 & 3 & 0 & 0 & 214 \\
\hline Scope of information presented & 139 & 70 & 5 & 0 & 0 & 214 \\
\hline Usefulness of the information & 138 & 68 & 8 & 0 & 0 & 214 \\
\hline Overall quality of the presentations & 134 & 74 & 6 & 0 & 0 & 214 \\
\hline Supporting resource materials & 131 & 69 & 13 & 1 & 0 & 214 \\
\hline Time allocated for networking & 126 & 69 & 19 & 0 & 0 & 214 \\
\hline $\begin{array}{l}\text { Amount of time dedicated to dis- } \\
\text { cussions in groups }\end{array}$ & 116 & 76 & 21 & 1 & 0 & 214 \\
\hline $\begin{array}{l}\text { Meetings' overall value in helping } \\
\text { you improve your professional ef- } \\
\text { fectiveness }\end{array}$ & 129 & 68 & 17 & 0 & 0 & 214 \\
\hline The number of participants & 121 & 70 & 21 & 2 & 0 & 214 \\
\hline $\begin{array}{l}\text { The workshop formula (e.g. two } \\
\text { half days) }\end{array}$ & 116 & 84 & 14 & 0 & 0 & 214 \\
\hline $\begin{array}{l}\text { Did the workshop cover what you } \\
\text { expected it to cover? }\end{array}$ & 126 & 74 & 12 & 1 & 0 & 213 \\
\hline Total & 1426 & 783 & 139 & 5 & 0 & 2353 \\
\hline
\end{tabular}

summarized needs of MoniQA experts. Again, the quality and integrity of information was not as good as expected because experts showed only little ambition in completing the requested data. Another problem was the discrepancy between small registration fees (resulting in lower financial income) and great travel costs for some training in Asia, when European experts were among the key speakers. Besides the financial aspect, finding appropriate trainers sometimes came to grief due to lack of time and interest of experts in preparing for and traveling to training. Although masked by the mobility scheme, the fact that especially younger experts simply could not afford attending training abroad without support from MoniQA could not be solved. International face to face training is only available at certain cost for the participant.

Sometimes one workshop attracted people from several different countries. Although all of them belonged to the same target group, huge differences in terms of pre-knowledge was observable and hence some participants were always underchallenged, and others were over-challenged. Besides a lack in technological skills, sometimes language barriers hampered the success of training. Organizational problems were mainly budget related due to unreasonable budgets and/or different opinions on registration fees. Minor problems arose from technical (internet access) or cultural (prayer rooms, special dishes) issues.

\section{Conclusions}

A global training network depends very much on the local support and because of different needs and different cultures it is very important to have written procedures and to communicate this to all concerned people. Especially the specification of learning outcomes was very important for the trainers to develop courses and for the participants to find the appropriate course. The choice of appropriate technologies is also very important. For example the multilingual user interface of the e-learning platform was very helpful. The implementation of a Quality Assurance system was difficult in terms of convincing all the partners to follow the rules and to complete the forms. But this will be very helpful to maintain the quality in future.

The training activities carried out were of high value in terms of bringing together scientists from different regions and cultures of the globe, involving highly qualified trainers as basis for a sustainable network in the future. Difficulties in financing travelling costs have been encountered, but this can be balanced by the use of e-learning and web seminars. The communication among 
Food Scientist Training |125

people from different cultures and the use of the internet and of different electronic systems poses some limitations, which sometimes need innovative solutions. As also other networks are carrying out training, a future challenge is to join forces, at least for activities like e-learning and web seminars.

In conclusion it can be stated, that the network was a driver for enhanced collaboration among partner organizations. Two partners for example, a University from Austria and a University from Vietnam, currently are in the preparatory phase of a new joint curricula in food safety. After 5 years of MoniQA training, the overall opinion on the programme is considered positive. Hence, it is expected that organizations which have been involved in the training programme are eager to contribute to any training in future. This increased ambition of several organizations all around the world in training activities might turn out as a durable impact.

\section{Acknowledgements}

FP6 project T5.4.5.1: Quality and safety control strategies for food (NOE) "MoniQA" (Contract No, FOOD-CT-2006-036337)

\section{References}

EC. (2000). White paper on food safety. Accessed: January, 2012, Retrieved from http: / / ec . europa . eu / dgs / health_consumer / library/pub/pub06_en.pdf

ENQA. (2009). Standards and Guidelines for Quality Assurance in the European Higher Education Area - 3rd editionRetrieved: January 19, 2012, ISBN 978-952-5539-24-0 (PDF). Retrieved from http://www.enqa. eu/pubs_esg.lasso

Hill B. (2010). The EU "Hygiene Package": its origins and implications for project countries and species. Accessed: January, 2012, Retrieved from http://seatglobal.eu/wpcontent/uploads/2010/02/8.-EU-hygienepackage-Barry-Hill.pdf
ISO 9001. (2008). Quality management systems - Requirements. International Organization for Standardization. CH-1211 Geneva 20, Switzerland.

MoniQA. (2009c). Bursaries - Eligibility and applications. January, 2012, Retrieved from http://www.moniqa.eu/node/888

MoniQA. (2009a). Deliverable 1.4 - Database of research infrastructure supplement with new features. Internal progress report. Unpublished.

MoniQA. (2008a). Deliverable 9.1 - Inventory of education/Training courses and offering institutions. Internal progress report, Unpublished.

MoniQA. (2012b). Deliverable 9.18 - Report on elearning activities. Internal progress report, Unpublished.

MoniQA. (2008b). Deliverable 9.2 - Analysis of available courses and gaps. Internal progress report. Unpublished.

MoniQA. (2012a). Deliverable 9.21 - Quality assurance system for training is tested and implemented. Internal progress report, Unpublished.

MoniQA. (2009b). Deliverable 9.5 - Inventory of external training courses and offering institutions. Internal progress report, Unpublished.

MoniQA. (2010a). Deliverable 9.7 - Two elearning courses are ready for testing. Internal progress report. Unpublished.

MoniQA. (2007a). EU funded project MoniQA - Towards the harmonisation of analytical methods for monitoring food quality and safety in the food supply chain. January, 2007, Retrieved from http://www.moniqa. eu / sites / moniqa . eu / files / pagefiles / MoniQA_presentation.pdf

MoniQA. (2007c). MoniQA activities - Joint resreach. January, 2007, Retrieved from http://www.moniqa.eu/node/228

MoniQA. (2007b). MoniQA activities - Working together. January, 2007, Retrieved from http://www.moniqa.eu/node/38

Salmon, G. (2002). E-tivities: the key to active online learning. Sterling, VA : Stylus Publishing Inc. 